\title{
Metodología de Medición del Potencial Exportador de las Organizaciones Empresariales
}

\author{
Efraín De La Hoz ${ }^{(1) *}$, Ángel L. González ${ }^{(2)}$ y Alfredo Santana ${ }^{(3)}$ \\ (1) Universidad de Cartagena, Facultad de Ciencias Económicas, Programa Administración Industrial. \\ Av. del Consulado, calle 30 No. 48-152, Cartagena - Colombia (e-mail: edelahozg@unicartagena.edu.co). \\ (2) Universidad del Norte, División de Ingenierías, Departamento de Ingeniería Industrial. Km. 5 vía \\ puerto, Barranquilla - Colombia (e-mail: agonzale@uninorte.edu.co). \\ (3) Tecnologico de Monterrey, campus Querétaro, División de Ingeniería y Arquitectura, Escuela de \\ Ingeniería Mecánica e Industrial. Epigmenio González 500.76130 Querétaro, Qro., México. \\ (e-mail: alfredo.santana@itesm.mx). \\ ${ }^{*}$ Autor a quien debe ser dirigida la correspondencia.
}

Recibido May. 16, 2016; Aceptado Jun. 18, 2016; Versión final Jul. 14, 2016, Publicado Dic. 2016

\begin{abstract}
Resumen
El presente trabajo expone una metodología de medición del potencial exportador de las organizaciones empresariales. Se desarrolló una revisión de la literatura relacionada con la orientación exportadora, para identificar factores clave en la competitividad exportadora. Con esta información se diseñó y validó un test de medición. Seguidamente, se plantearon métricas para la valoración competitiva exportadora. Luego se expone una aplicación en un sector empresarial. Los resultados muestran 16 factores clave integrados en las dimensiones financiera, procesos internos, clientes, aprendizaje y crecimiento y mercados. Esto está validado mediante un índice de validez de contenido de 0.91 y un coeficiente de confiabilidad Alfa de Cronbach de 0,97. Basados en los resultados del trabajo, se concluye que la metodología presentada es capaz de medir las condiciones competitivas exportadoras de las empresas con lo cual se pueden identificar y priorizar oportunidades de mejora.
\end{abstract}

Palabras clave: potencial exportador; competitividad; comercio exterior; validez de contenido; confiabilidad.

\section{Methodology for Measuring the Potential Exporter of Business Organizations}

\begin{abstract}
This paper presents a methodology for measuring the export potential of business organizations. The literature related to orientation exporting was reviewed to identify the key factors in export competitiveness. With this information a measurement test was designed and validated. After this, metrics for the evaluation of export competitivity were defined. Following this, an application of the methodology in a business sector was done. The results show 16 key factors integrated in the financial, internal processes, customers, learning and growth, and markets. These results are validated by a content validity rate of 0.91 and a reliability coefficient Cronbach's alpha of 0.97 . Based on the results of this work, it is concluded that the proposed methodology is capable of measuring export competitive conditions for enterprises which can identify and prioritize opportunities for improvement
\end{abstract}

Keywords: export potential; competitiveness; foreign trade; content validity; reliability 


\section{INTRODUCCIÓN}

Diversos autores han abordado el tema del potencial exportador intentando establecer condiciones favorables para lograr el posicionamiento de las organizaciones en el comercio internacional. En este sentido, Escandón y Hurtado (2014a) identifican factores determinantes para el desarrollo temprano de procesos de internacionalización de pequeñas y medianas empresas. Así mismo, Navarro, Arenas y Rondán (2014), evalúan el impacto del entorno en el comportamiento estratégico, a partir de variables asociadas a la turbulencia de mercados y tecnología, distancias psicológicas entre los mercados nacional e internacional y la capacidad adaptativa. Vinculan la orientación al mercado como factor de apoyo en la toma de decisiones estratégicas, contribuyendo directa y positivamente en los resultados de exportación.

Por su parte, Cabrera y Olivares (2012), estudian la influencia de factores como el capital humano, el capital social y los recursos financieros en la velocidad con que se desarrolla el proceso de exportación de las empresas familiares. Akdeve (2013) analiza la competitividad en el mercado nacional e internacional, sus resultados muestran una relación positiva del tamaño de la empresa, la edad y el nivel de los estándares de calidad en el potencial de exportación. Cabarcas y Paternina (2011), concluyen que existen diferencias significativas en los perfiles productivos de las empresas exportadoras y no exportadoras. Adicionalmente, autores como Kohli y Jaworski (1990), Rose y Shoham (2002), Madrid y García (2004), Escandón y Hurtado (2014b), proponen aspectos y variables para la medición de las condiciones competitivas de las empresas en un contexto de comercio internacional.

En la actualidad los mercados son cada vez más cambiantes y competitivos, siendo necesario los procesos de medición de indicadores para evaluar la eficiencia de la empresa (Matilla y Chalmeta, 2007), De esta manera, es importante que las empresas midan y evalúen los factores clave que potencializan sus condiciones para competir con probabilidades de éxito en los mercados internacionales. La literatura muestra trabajos relacionados con la competitividad y la orientación al mercado, sin embargo las investigaciones relacionadas con los factores que afectan los resultados de exportación en las organizaciones, es muy fragmentada, con frecuencia contradictoria y analizan desde múltiples dimensiones el desempeño de las organizaciones en las exportaciones.

En este trabajo, se estructura una metodología para medir el potencial exportador de las organizaciones, para lo cual se identificaron factores clave para la competitividad exportadora, se diseñó y validó un cuestionario con factores clave de competitividad exportadora y se definieron las métricas para la valoración competitiva. Finalmente, se hace una aplicación en un sector empresarial como soporte a la metodología.

\section{METODOLOGÍA}

La presente investigación se desarrolló bajo dos enfoques, un enfoque experimental dado que contempla la validación y aplicación de un instrumento de medición y el análisis de resultados en empresas de un sector empresarial, y un enfoque racional a partir de un proceso de análisis y construcción de conocimiento soportado en argumentos teóricos y experimentales. Razón por la cual el origen del conocimiento se sustenta en una concepción empirista y racionalista de la ciencia. Para la identificación de los factores clave de competitividad exportadora, se hizo una revisión de la literatura relacionada con el análisis de métricas para el comercio internacional en la que se identificaron las variables propuestas por diversos autores. En la Tabla 1, se sintetizaron en 5 dimensiones los aportes de diversos autores tomando como marco el modelo de gestión de Kaplan y Norton (2004), agregando la dimensión mercado por su relevancia especial en el análisis competitivo exportador.

Seguidamente, en la Tabla 2 se sistematizaron 16 factores clave en la medición del potencial exportador y se establecieron sus objetivos, los cuales orientan en la construcción del instrumento de medición. Se diseñó el instrumento de medición incorporando ítems asociados a cada uno de los 16 factores clave y se evaluó su validez de contenido, así como su confiabilidad y consistencia interna.

La validez de contenido es una prueba que se realiza con el objeto de establecer que tan adecuado es un instrumento para medir lo que se pretende, a partir de un muestreo realizado de un universo de posibles ítems. Para su estimación generalmente es utilizada la Razón de Validez de Contenido CVR (Content Validity Ratio) de Lawshe (Cohen y Swerdlik, 2001). Lawshe (1975), plantea la conformación de un panel de especialistas en la actividad a evaluar, los cuales emitirán juicio de cada ítem que conforma el instrumento bajo las categorías de: esencial, útil pero no esencial y no necesario. Seguidamente, se establece el número de acuerdos en la categoría "esencial" para lo cual Lawshe propone la CVR para cada ítem, definida como: 
$C V R=\frac{\mathrm{n}_{\mathrm{e}}-\frac{N}{2}}{\frac{N}{2}}$

Siendo: $\mathrm{n}_{\mathrm{e}}$ : Número de jueces con acuerdo en la categoría esencial, y $\mathrm{N}$ : Número total de jueces o panelistas. La expresión anterior se establece como una correlación en el nivel de acuerdo de los jueces en la categoría "esencial", por tomar valores entre -1 y 1.

Tabla 1. Variables asociadas a la competitividad exportadora

\begin{tabular}{|c|c|c|c|c|c|}
\hline Autor & Financiera & Procesos Internos & $\begin{array}{l}\text { Aprendizaje y } \\
\text { Crecimiento }\end{array}$ & Clientes & Mercado \\
\hline $\begin{array}{l}\text { Kohli y } \\
\text { Jaworski } \\
\text { (1990) }\end{array}$ & Rentabilidad & Capacidad de respuesta. & Difusión de Inteligencia & $\begin{array}{l}\text { Enfoque al } \\
\text { cliente }\end{array}$ & $\begin{array}{l}\text { Inteligencia de } \\
\text { Mercado, } \\
\text { coordinación } \\
\text { de marketing }\end{array}$ \\
\hline $\begin{array}{l}\text { Rose y } \\
\text { Shoham } \\
(2002\end{array}$ & $\begin{array}{l}\text { Ventas de export., } \\
\text { beneficios } \quad \text { de } \\
\text { export., cambio en } \\
\text { export., ganancias } \\
\text { de export. }\end{array}$ & $\begin{array}{l}\text { Capacidad de respuesta, } \\
\text { intensidad competitiva }\end{array}$ & Difusión de Inteligencia & & $\begin{array}{l}\text { Generación de } \\
\text { inteligencia, } \\
\text { turbulencia de } \\
\text { los mercados } \\
\text { y tecnología }\end{array}$ \\
\hline $\begin{array}{l}\text { Madrid y } \\
\text { García } \\
(2004)\end{array}$ & $\begin{array}{l}\text { Oportunidades de } \\
\text { crecimiento, } \\
\text { productividad del } \\
\text { trabajo, rentabilidad } \\
\end{array}$ & Tamaño de la empresa & $\begin{array}{l}\text { Características de la } \\
\text { dirección (Formación } \\
\text { del gerente), experiencia } \\
\text { en exportación }\end{array}$ & $\begin{array}{l}\text { Tamaño del } \\
\text { mercado }\end{array}$ & \\
\hline $\begin{array}{l}\text { Harms } \\
(2009)\end{array}$ & & $\begin{array}{l}\text { Características de la alta } \\
\text { dirección }\end{array}$ & & $\begin{array}{l}\text { Tipo de } \\
\text { industria }\end{array}$ & \\
\hline $\begin{array}{l}\text { Cabarcas } \\
\text { y } \\
\text { Paternina } \\
(2011)\end{array}$ & & $\begin{array}{l}\text { Porcentaje de utilización de la } \\
\text { maquinaria, cantidad de } \\
\text { materia prima importada, } \\
\text { porcentaje de facturas con } \\
\text { inconsistencias, número de } \\
\text { accidentes de trabajo, } \\
\text { implementación de sistemas } \\
\text { de gestión de la calidad y } \\
\text { sistemas de gestión ambiental }\end{array}$ & $\begin{array}{l}\text { Porcentaje } \\
\text { empleados capacitados }\end{array}$ & & \\
\hline $\begin{array}{l}\text { Cabrera y } \\
\text { Olivares } \\
\text { (2012) } \\
\end{array}$ & $\begin{array}{l}\text { Recursos } \\
\text { financieros }\end{array}$ & & & $\begin{array}{l}\text { Capital } \\
\text { humano }\end{array}$ & Capital social. \\
\hline $\begin{array}{l}\text { Akdev } \\
(2013)\end{array}$ & & Tamaño, edad. & & & $\begin{array}{l}\text { Nivel de los } \\
\text { estándares de } \\
\text { calidad }\end{array}$ \\
\hline $\begin{array}{l}\text { Fernández } \\
\text {, et al } \\
\text { (2014) }\end{array}$ & & $\begin{array}{l}\text { Dimensión de la empresa, } \\
\text { productividad, sofisticación de } \\
\text { la empresa }\end{array}$ & & $\begin{array}{l}\text { Condiciones } \\
\text { del } \\
\text { emprendedor }\end{array}$ & \\
\hline $\begin{array}{l}\text { Escandón } \\
\text { y Hurtado } \\
\text { (2014) }\end{array}$ & $\begin{array}{l}\text { Tamaño de las } \\
\text { ventas, intensidad } \\
\text { exportadora de la } \\
\text { empresa, } \\
\text { disponibilidad de } \\
\text { recursos, fortalezas }\end{array}$ & $\begin{array}{l}\text { Capacidad Innovadora de la } \\
\text { empresa, inversión en I+D. } \\
\text { Innovación producto, modo de } \\
\text { expansión, localización } \\
\text { geográfica, nivel tecnológico, } \\
\text { factores internos, acciones en } \\
\text { innovación }\end{array}$ & $\begin{array}{l}\text { Expectativas de } \\
\text { crecimiento y } \\
\text { generación de empleo, } \\
\text { actividad emprendedora, } \\
\text { edad del emprendedor, } \\
\text { formación, experiencia, } \\
\text { actitud al riesgo }\end{array}$ & $\begin{array}{l}\text { Percepción de } \\
\text { oportunidades }\end{array}$ & $\begin{array}{l}\text { Innovación, } \\
\text { actividad, } \\
\text { intensidad } \\
\text { export. del } \\
\text { sector, } \\
\text { características } \\
\text { del mercado }\end{array}$ \\
\hline
\end{tabular}

Tabla 2. Factores clave en la medición del potencial exportador

\begin{tabular}{|l|l|l|}
\hline Dimensión & \multicolumn{1}{|c|}{ Factores } & \multicolumn{1}{c|}{ Objetivo } \\
\hline \multirow{4}{*}{ Financiera } & Gestión Financiera (F1) & Evaluar resultados financieros \\
\cline { 2 - 3 } & Gestión del Riesgo (F2) & Evaluar diversificación \\
\cline { 2 - 3 } & Salud Financiera (F3) & Evaluar liquidez. \\
\hline \multirow{4}{*}{ Mercado } & Conocimiento del Mercado (M1) & Identificar oportunidades y amenazas \\
\cline { 2 - 3 } & Gomercio exterior (M2) & Conocer condiciones del comercio internacional \\
\cline { 2 - 3 } yprendizaje & Competitividad del Producto (M3) & Conocer condiciones del producto en el mercado internacional \\
\cline { 2 - 3 } Crecimiento & Gestión de la Información (AC1) & Evaluar flujo de información \\
\cline { 2 - 3 } & Gestión del Conocimiento(AC2) & Evaluar la gestión de capacitación y competencias \\
\hline \multirow{3}{*}{ Clientes } & Gestión de Clima Laboral (AC3) & Analizar condiciones colaborativas internas \\
\cline { 2 - 3 } & Gestión de Proveedores (C1) & Analizar colación con proveedores \\
\cline { 2 - 3 } & Gestión de Clientes (C2) & Analizar satisfacción del servicio \\
\hline \multirow{3}{*}{\begin{tabular}{l} 
Procesos Internos \\
\cline { 2 - 3 }
\end{tabular}} & Gestión de Productividad (PI1) & Evaluar acciones de mejoramiento del servicio \\
\cline { 2 - 3 } & Gestión de Innovación (PI2) & Analizar resultados de operaciones productivas \\
\cline { 2 - 3 } & Factores Logísticos (PI3) & Evaluar procesos de investigación y desarrollo \\
\cline { 2 - 3 } & G. de Tec. y Operaciones (PI4) & Evaluar condiciones de tecnología \\
\hline
\end{tabular}


Con la CVR de cada ítem, se calcula el Índice de Validez de Contenido CVI (Content Validity Index) de todo el instrumento o formulario, definido por:

$C V I=\frac{\sum_{i=1}^{M} C V R_{i}}{M}$

Donde $C V R_{i}$ : Razón de Validez de Contenido de los ítems que cumplen con el valor mínimo establecido por Lawshe según el número de jueces y $\mathrm{M}$ el total de ítems aceptados en la prueba.

Adicionalmente, Tristán (2008) plantea eliminar el efecto del número de jueces sobre los valores de referencia. Su análisis establece como condición normalizada para que un instrumento o banco de ítems tenga validez un $\mathrm{CVR}^{\prime} \geq 0.5823$ y $\mathrm{CVI}^{\prime} \geq 0.5823$.

Para analizar la fiabilidad, se utilizó el coeficiente Alfa de Cronbach ( $\alpha$ ), el cual es una medida de la consistencia interna, que depende de las correlaciones entre todos los ítems de un cuestionario (Soler, 2008). Para su cálculo se utilizó la relación:

$\alpha=\frac{k}{k-1}\left[1-\frac{\sum_{i=1}^{k} \sigma^{2}(i)}{\sigma^{2}(X)}\right]$

Donde $i$ son los ítems del cuestionario, $k$ es el número de ítems, $\sigma^{2}(X)$ es la varianza del total de las observaciones, $\sigma^{2}(i)$ es la varianza del ítem $i$.

Autores como George y Mallery (2003) conceptúan que un coeficiente de alfa de Cronbach $>0.9$ indica una excelente fiabilidad del instrumento, $>0.8$ indica una buena fiabilidad, $>0.7$ es aceptable, $>0.6$ es cuestionable, $\leq 0.6$ es pobre o inaceptable. Sin embargo, Gliem y Gliem (2003) afirman que un valor superior a 0.8 es una meta razonable.

Finalmente, la valoración competitiva define el nivel competitivo de la organización, se expresa mediante la puntuación promedio de los ítems en el factor, la puntuación promedio obtenida en cada dimensión $D_{i}$ y la puntuación promedio total $P$ del potencial exportador de la empresa dada por:

$D_{i}=\frac{\sum_{j=1}^{k} X_{i j}}{P_{\max -i}} * 100$

Donde $X_{i j}$ representa el puntaje obtenido en cada ítem j de la dimensión i, $P_{\max -i}$ el puntaje máximo posible de la dimensión i y $\mathrm{k}$ el número de ítems en la dimensión i.

$P=\frac{\sum_{i=1}^{n} \sum_{j=1}^{k} X_{i j}}{\sum_{i=1}^{n} P_{\max -i}} * 100$

Siendo $P$ una medida porcentual comparable con el nivel ideal del $100 \%, n$ es el número de dimensiones y $P_{\max }$ es la suma de los puntajes máximos de cada dimensión i, cuando cada ítem es calificado con un nivel alto. Estos indicadores permiten evaluar las organizaciones e identificar oportunidades de mejora.

\section{RESULTADOS Y DISCUSIÓN}

A partir de los factores clave, se diseñó el instrumento MFC_PE (Medición de Factores Clave del Potencial Exportador), para medir el nivel competitivo exportador de las empresas, el cual se presenta en la Tabla 3. Se establecieron entre 3 y 4 ítems para cada factor del modelo obteniéndose un total de 61 ítems, los cuales se sometieron a validez de contenido mediante juicio de expertos y pruebas de fiabilidad y consistencia interna. Para efectos de cuantificar los resultados del instrumento MFC_PE, se definió una escala de 0 a 3, la cual refleja la valoración de cada ítem en las categorías nulo (0), bajo (1), medio (2) y alto (3).

En la Tabla 4, se presentan los resultados de la evaluación de contenido de los factores para lo cual seleccionaron 8 expertos atendiendo las recomendaciones de Skjong y Wentworht (2000). Se observa validez de contenido del instrumento dado que las CVR' de los factores clave de competitividad exportadora y el CVI' $(0,91)$ del instrumento son mayores a 0.58 , con lo cual se cumple con el criterio de validez expuesto en el modelo de Tristán. 


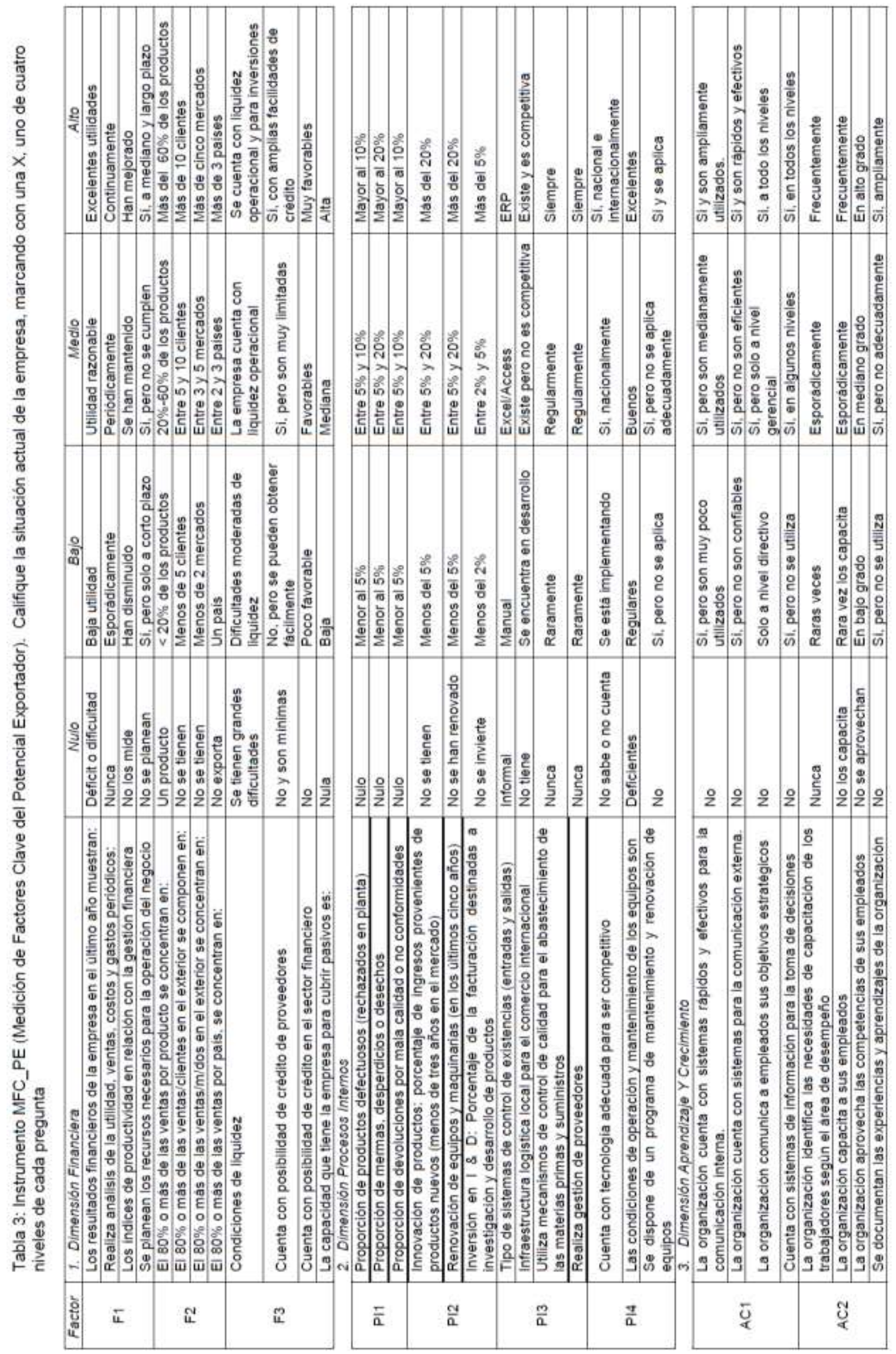




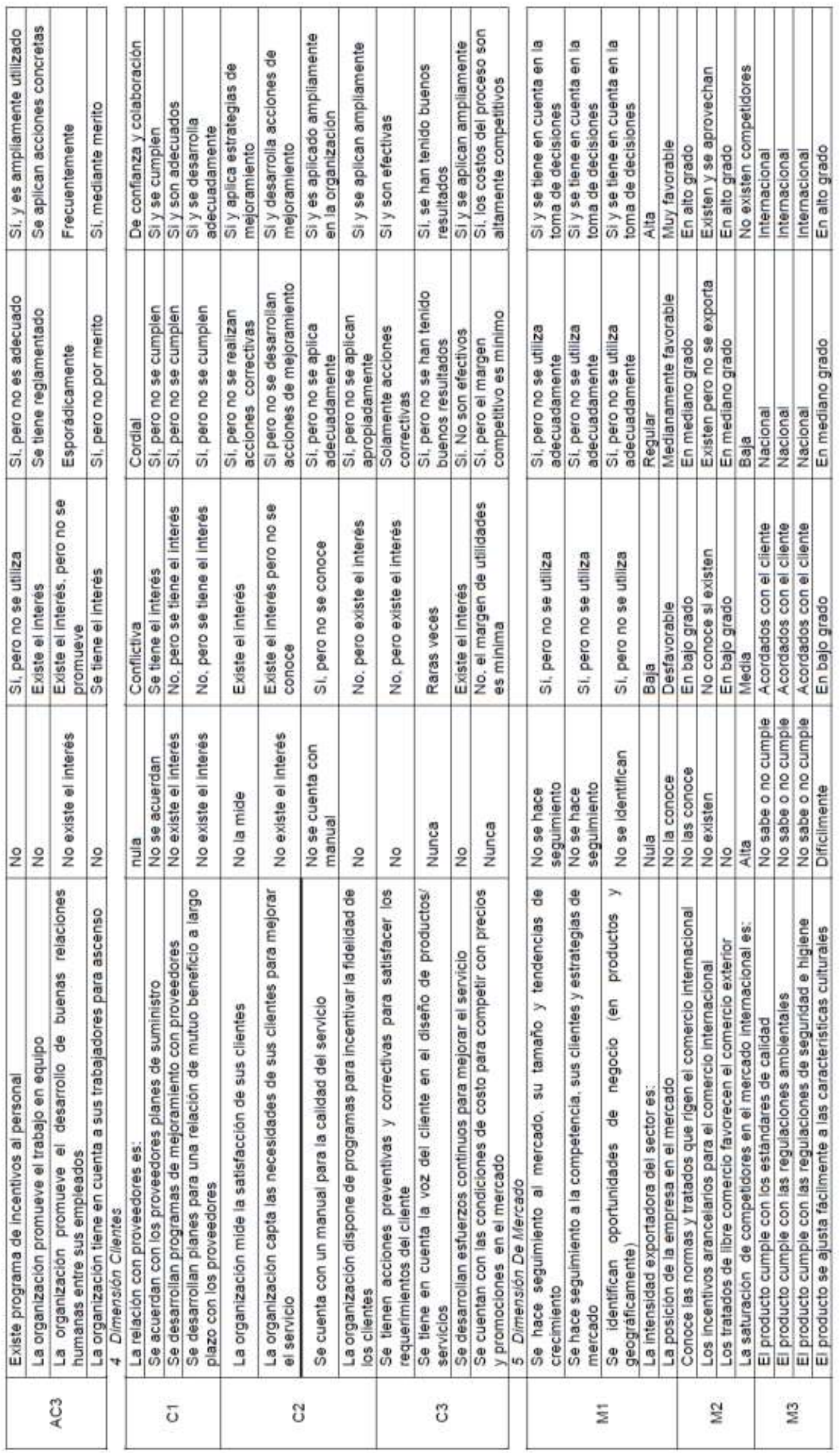


Tabla 4. Resultados validez de contenido de factores

\begin{tabular}{|c|l|c|}
\hline Dimensión & \multicolumn{1}{|c|}{ Factores } & CVR' \\
\hline \multirow{3}{*}{ Financiera } & Gestión Financiera & 0,88 \\
\cline { 2 - 3 } & Gestión del Riesgo & 0,97 \\
\cline { 2 - 3 } & Salud Financiera & 0,85 \\
\hline \multirow{3}{*}{$\begin{array}{c}\text { Procesos } \\
\text { Internos }\end{array}$} & Gestión de Productividad & 0,92 \\
\cline { 2 - 3 } & Gestión de Innovación & 0,92 \\
\cline { 2 - 3 } & Factores Logísticos & 0,85 \\
\cline { 2 - 3 } & Gestión de Tecnologías y Operaciones & 1,00 \\
\hline $\begin{array}{c}\text { Aprendizaje y } \\
\text { Crecimiento }\end{array}$ & Gestión de Información & 1,00 \\
\cline { 2 - 3 } & Gestión del Conocimiento & 0,90 \\
\hline
\end{tabular}

Continuación
\begin{tabular}{|l|l|c|}
\hline Dimensión & \multicolumn{1}{|c|}{ Factores } & CVR' \\
\hline & Gestión del Clima Laboral & 0,82 \\
\hline \multirow{4}{*}{ Clientes } & Gestión de Proveedores & 0,94 \\
\cline { 2 - 3 } & Gestión de Clientes & 0,91 \\
\cline { 2 - 3 } & Gestión de Requerimientos & 0,85 \\
\hline \multirow{3}{*}{ Mercado } & Conocimiento del Mercado & 0,94 \\
\cline { 2 - 3 } & Comercio Exterior & 0,91 \\
\cline { 2 - 3 } & Competitividad del Producto & 0,94 \\
\hline \multicolumn{1}{|c|}{ Índice de Validez de Contenido (CVI) } & 0,91 \\
\hline
\end{tabular}

La confiabilidad del instrumento, se evaluó mediante una muestra de 20 empresas del Sector Químico del Departamento del Atlántico, Colombia. Los resultados de la Tabla 5 muestran confiabilidad mínima de "aceptable" por factor y excelente del instrumento MFC_PE al verificarse un valor de 0,98 , superior al 0,9 planteado por George y Mallery (2003).

Tabla 5. Coeficiente Alfa de Cronbach por dimensión y factores del Instrumento MFC_PE

\begin{tabular}{|c|c|l|c|}
\hline Dimensión & $\alpha$ & \multicolumn{1}{|c|}{ Factor } & $\alpha$ \\
\hline \multirow{4}{*}{ Financiera } & 0,90 & Gestión Financiera & 0,79 \\
\cline { 3 - 4 } & & Gestión del Riesgo & 0,89 \\
\cline { 3 - 4 } Mercado & Salud Financiera & 0,77 \\
\hline \multirow{2}{*}{$\begin{array}{c}\text { Aprendizaj } \\
\text { e y } \\
\text { Crecimient } \\
\text { o }\end{array}$} & 0,93 & Conocimiento del Mercado & 0,93 \\
\cline { 3 - 4 } & & Comercio exterior & 0,88 \\
\cline { 3 - 4 } & & Competitividad del Producto & 0,82 \\
\cline { 3 - 4 } & Gestión de la Información & 0,78 \\
\cline { 3 - 4 } & Gestión del Conocimiento & 0,82 \\
\hline
\end{tabular}

\begin{tabular}{|c|c|c|c|}
\hline Dimensión & $\alpha$ & Factor & $\alpha$ \\
\hline \multirow{3}{*}{ Clientes } & \multirow{3}{*}{0,92} & Gestión de Proveedores & 0,80 \\
\hline & & Gestión de Clientes & 0,88 \\
\hline & & $\begin{array}{l}\text { Gestión de } \\
\text { Requerimientos }\end{array}$ & 0,81 \\
\hline \multirow{4}{*}{$\begin{array}{l}\text { Procesos } \\
\text { Internos }\end{array}$} & \multirow{4}{*}{0,86} & Gestión de Productividad & 0,71 \\
\hline & & Gestión de Innovación & 0,77 \\
\hline & & Factores Logísticos & 0,82 \\
\hline & & $\begin{array}{l}\text { Gestión de Tecnologías y } \\
\text { Operaciones }\end{array}$ & 0,72 \\
\hline \multicolumn{3}{|c|}{$\alpha$ (del Instrumento) } & 0,98 \\
\hline
\end{tabular}

Seguidamente, se aplicó el instrumento a 3 empresas del sector químico, con el objeto de valorar el nivel competitivo del potencial exportador. En la Tabla 6 se presentan los resultados promedios por factor y la valoración competitiva por empresa y dimensión, en la que se subrayan los factores clave con oportunidades de mejora comparados con su valoración competitiva promedio.

Tabla 6. Valoración competitiva de empresas en el sector químico

\begin{tabular}{|c|c|c|c|c|c|c|c|c|c|c|c|c|c|c|c|c|c|}
\hline \multirow{2}{*}{ Empresa } & \multicolumn{3}{|c|}{ Financiera } & \multicolumn{4}{|c|}{ Procesos Internos } & \multicolumn{3}{|c|}{$\begin{array}{l}\text { Aprendizaje. y } \\
\text { Crecimiento }\end{array}$} & \multicolumn{3}{|c|}{ Clientes } & \multicolumn{3}{|c|}{ Mercado } & \multirow{2}{*}{ Promedio } \\
\hline & F1 & F2 & F3 & $\mathrm{Pl1}$ & $\mathrm{PI} 2$ & $\mathrm{PI} 3$ & $\mathrm{PI} 4$ & $\mathrm{AC1}$ & AC2 & AC3 & $\mathrm{C} 1$ & C2 & C3 & M1 & M2 & $\mathrm{M} 3$ & \\
\hline \multirow{2}{*}{1} & 1,25 & 0,50 & $\underline{0,00}$ & $\underline{0,67}$ & 0,33 & $\underline{\underline{0}, 50}$ & 1,33 & 1,75 & 1,75 & 1,00 & $\underline{0,75}$ & 1,25 & 1,25 & $\underline{0,80}$ & 0,33 & 2,00 & \multirow{2}{*}{0,97} \\
\hline & \multicolumn{3}{|c|}{0,58} & \multicolumn{4}{|c|}{0,71} & \multicolumn{3}{|c|}{1,50} & \multicolumn{3}{|c|}{1,08} & \multicolumn{3}{|c|}{1,04} & \\
\hline \multirow{2}{*}{2} & 1,00 & 1,00 & 1,00 & $\underline{0,67}$ & 1,67 & 2,50 & 2,67 & 2,25 & 2,25 & 2,25 & 2,50 & 2,50 & 2,50 & 1,80 & 1,67 & 2,00 & \multirow{2}{*}{1,89} \\
\hline & & 1,00 & & & & 88 & & & 2,25 & & & 2,50 & & & 1,82 & & \\
\hline \multirow[t]{2}{*}{3} & 3,00 & 2,50 & 2,75 & 1,00 & 1,67 & 3,00 & 2,67 & 3,00 & 3,00 & 3,00 & 3,00 & 3,00 & 3,00 & 2,80 & 2,67 & 2,50 & \multirow{2}{*}{2,66} \\
\hline & & 2,75 & & & & 08 & & & 3,00 & & & 3,00 & & & 2,66 & & \\
\hline
\end{tabular}

\section{CONCLUSIONES}

De los resultados de la investigación, se puede inferir que los factores clave propuestos en las dimensiones Financiera, Procesos Internos, Aprendizaje y crecimiento, Clientes y Mercado de la metodología, son válidos para evaluar el potencial exportador. Así mismo, el cuestionario propuesto, es altamente confiable y válido para evaluar el potencial exportador de las organizaciones empresariales, superando resultados de investigaciones previas.

La perspectiva adoptada en nuestro trabajo difiere en una serie de aspectos con estudios previos de la orientación exportadora. Primero, metodológicamente se hizo un inventario de trabajos relacionados con la medición del potencial exportador y la orientación exportadora en el que se identificaron factores clave. Segundo, se sistematiza e integran estos factores, desde un enfoque estratégico de manera que se intenta conciliar los planteamientos realizados desde perspectivas diferentes. Tercero, el método planteado es exhaustivo en el análisis de las métricas para evaluar condiciones competitivas para el comercio exterior. Finalmente, la metodología presentada en este trabajo es aplicable a distintos sectores y contextos de 
mercados empresariales y puede ser complementada con metodologías para evaluar los resultados de indicadores financiero (De La Hoz, et al., 2014; Fontalvo et al., 2012; Herrera et al., 2012).

\section{REFERENCIAS}

Akdeve, E., The determinants of export potential: A case of Ankara manufacturing sector. http://www.ijbmer.com/docs/volumes/vol4issue3/ijbmer2013040304.pdf, ISSN: 2229-6247, International J. of Business Management \& Economic Research, 4(3), 745-751. (2013)

Cabarcas, J., y Paternina, C., Aplicación del análisis discriminante para identificar diferencias en el perfil productivo de las empresas exportadoras y no exportadoras del Departamento del Atlántico de Colombia. ingeniare, ISSN: 1909-2458, Ingeniare, Universidad Libre-Barranquilla, 6(10), 33-48. (2011)

Cabrera, K., y Olivares, A., La influencia de los recursos de capital humano, social y financiero sobre la velocidad del proceso exportador de las empresas familiares, doi: 10.1016/j.redee.2012.07.003, Rev. Europea de Dirección y Economía de La Empresa, 21(4), 306-315. (2012)

Cohen, R., y Swerdlik, M., Pruebas y evaluación psicológicas: Introducción a las pruebas y a la medición. (4a ed.). México: Mc.Graw Hill. (2001)

De La Hoz, E., Fontalvo, T., y Morelos, J. Evaluación del comportamiento de los indicadores de productividad y rentabilidad financiera del sector petróleo y gas en Colombia mediante el análisis discriminante, doi: 10.1016/S0186-1042(14)70159-7, Contaduría y Administración, 59(4), 167-191. (2014)

Escandón, D., y Hurtado, A., Factores que influyen en el desarrollo exportador de las pymes en Colombia, doi: 10.1016/j.estger.2014.04.006, Estudios Gerenciales, 30(118), 172-183. (2014a)

Escandón, D., y Hurtado, A., Los determinantes de la orientación exportadora y los resultados en las pymes exportadoras en Colombia, doil: 10.1016/j.estger.2014.05.002, Est. Gerenciales, 30(133), 430-440. (2014b)

Fontalvo, T., Vergara, J., y De La Hoz, E. Evaluación del mejoramiento de los indicadores financieros en las empresas del sector almacenamiento y actividades conexas en Colombia por medio de análisis de discriminante, http://repositorio.uac.edu.co/handle/11619/1259, Prospectiva, 10(1), 124-131. (2012)

Fontalvo, T, Gómez, J., y De La Hoz, E. Aplicación de análisis discriminante para evaluar el comportamiento de los indicadores financieros en las empresas del sector carbón en Colombia, http://www.scielo.org.co/pdf/entra/v8n2/v8n2a05.pdf, Revista Entramado, 8(2), 64-73. (2012)

George, D., y Mallery, P., SPSS for Windows step by step: A Simple Guide and Reference (4a ed.). Boston (2003)

Gliem, J. a, y Gliem, R. R., Calculating, Interpreting, and Reporting Cronbach's Alpha Reliability Coefficient for Likert-Type Scales,. In 2003 Midwest Research to Practice Conference in Adult, Continuing, and Community Education (pp. 82-88). (2003)

Kaplan, R., y Norton, D., Mapas Estratégicos. Convirtiendo los activos intangibles en resultados (pp. 1-500). Barcelona: Gestión 2000. (2004)

Kohli, A. K., y Jaworski, B. J. The Market orientation. Journal of Marketing, 54(2), 1-18. (1990)

Lawshe, H., A quantitative approach to content validity, doi: 10.1111/j.1744-6570.1975.tb01393.x, Personnel Psychology, 28(4), 563-575. (1975)

Matilla, M., y Chalmeta, R., Metodología para la implantación de un sistema de medición del rendimiento empresarial, doi: 10.4067/S0718-07642007000100016, Inf. Tecnológica. 18(1), 119-126. (2007)

Navarro, A., Arenas, J., y Rondán, J., External environment and the moderating role of export market orientation, doi:10.1016/j.jbusres.2013.11.037, Journal of Business Research, 67(5), 740-745. (2014)

Rose, G. M., y Shoham, A., Export performance and market orientation: Establishing an empirical link, doi: 10.1016/S0148-2963(00)00139-9, Journal of Business Research, 55(3), 217-225. (2002)

Skjong, R., y Wentworth, B., Expert Judgement and Risk Perception. Det Norske Veritas, 1-8. (2000)

Soler, S. Reliability coefficient of written tools in the frame of the classical theory of tests, Revista Cubana de Educación Médica Superior, 22(2), p1-14. (2008)

Tristán, A. Modificación al modelo de Lawshe para el dictamen cuantitativo de la validez de contenido de un instrumento objetivo, Avances en medición, 6, 37-48. (2008) 